\title{
Alveolar nitric oxide in adults with asthma: evidence of distal lung inflammation in refractory asthma
}

\author{
M. Berry, B. Hargadon, A. Morgan, M. Shelley, J. Richter, D. Shaw, R.H. Green, \\ C. Brightling, A.J. Wardlaw and I.D. Pavord
}

ABSTRACT: Recent studies have suggested that alveolar nitric oxide (NO) concentration is a noninvasive test of distal lung inflammation.

The current study determined whether alveolar NO concentration can be measured in patients with asthma of varying severity, tested the hypothesis that there is an association between alveolar NO and bronchoalveolar lavage (BAL) eosinophil count and determined whether refractory asthma is characterised by a raised alveolar NO concentration. Finally, the present authors assessed the effect of 2 weeks of prednisolone (30 $\mathrm{mg}$ q.d.) on alveolar NO concentration.

Alveolar NO concentration was both measurable and repeatable in patients with refractory asthma. A positive correlation was found between alveolar NO concentration and BAL eosinophil count but not with bronchial wash or sputum eosinophil count. Alveolar NO concentration was increased in patients with refractory asthma (7.1 ppb) compared with mildto-moderate asthma (3.4 ppb) and normal controls (3.4 ppb) and reduced by treatment with prednisolone.

In conclusion, these findings support the hypothesis that alveolar nitric oxide is a measure of distal airway inflammation and suggest that distal lung inflammation is present in refractory asthma.

KEYWORDS: Asthma, asthma mechanisms, asthma severity

$\mathbf{R}$ efractory asthma can be defined as asthma that cannot be controlled satisfactorily with inhaled corticosteroids [1]. It is present in $5 \%$ of a random population of patients with asthma and the majority of patients attending specialist respiratory clinics. Patients with refractory asthma suffer considerable morbidity and consume a large proportion of the health budget attributed to asthma management, on average six times more per patient than mild asthma. The American Thoracic Society (ATS) [1] and the European Respiratory Society [2] have identified research into the mechanisms of refractory asthma as an important priority.

An attractive hypothesis for the pathogenesis of refractory asthma is the presence of inflammation in the distal lung, an area that might not be accessed by inhaled corticosteroids. Post mortem and bronchoscopy with transbronchial biopsy studies have shown inflammation in the alveoli and small airways, although these studies were uncontrolled so it is not possible to determine whether this feature is peculiar to refractory asthma [3].

Airway inflammation can be measured noninvasively by measuring the sputum eosinophil count and single flow exhaled nitric oxide (NO) concentration, although these tests are limited to sampling the proximal airway. However, two compartment models of pulmonary $\mathrm{NO}$ production have been described $[4,5]$, which can be used to calculate the alveolar contribution to exhaled $\mathrm{NO}$ concentration. Alveolar NO concentration is elevated in conditions associated with distal lung inflammation, such as pulmonary fibrosis [6] and chronic obstructive pulmonary disease [7]. Unlike bronchial NO, alveolar NO production is not reduced by inhaled fluticasone in patients with asthma suggesting that it may be derived from a site not accessed by inhaled corticosteroid treatment [8]. Other methods that might be useful in the assessment of distal lung inflammation include bronchoalveolar lavage (BAL) [9] or transbronchial biopsy [10], although these methods are invasive and their application to

\section{AFFILIATIONS}

Dept of Respiratory Medicine and Thoracic Surgery, Institute for Lung Health, Glenfield Hospital, Leicester, UK.

CORRESPONDENCE

M. Berry

Dept of Respiratory Medicine and

Thoracic Surgery

Institute for Lung Health

Glenfield Hospital

Groby Road

Leicester LE3 9QP

UK

Fax: 441162367768

E-mail: mike_berry@blueyonder.

co.uk

Received:

November 192004

Accepted after revision:

March 112005

SUPPORT STATEMENT

This study was funded by Asthma UK. Asthma UK had no role in design, recruitment or analysis of data for this project. Flow independent NO analysis equipment was a generous gift from Aerocrine, Stockholm, Sweden, who also had no role in the study design, recruitment or collection and analysis of data.

Online ISSN 1399-3003 
patients with refractory asthma is limited. Alveolar NO concentration has been demonstrated to be associated with BAL eosinophilic cationic protein concentration in children with steroid-treated atopic asthma [11], but whether it is possible to measure alveolar NO in patients with refractory asthma, and how alveolar NO relates to other measurements of distal inflammation, is unknown.

The present authors have determined whether it is possible to measure alveolar $\mathrm{NO}$ concentration in patients with refractory asthma and examined repeatability. The hypotheses that alveolar NO concentration is associated with distal lung inflammation (as reflected by BAL eosinophil count) and that increased alveolar NO concentration is a feature of refractory asthma were tested. Finally, the effect of treatment with oral corticosteroids and higher dose inhaled steroids on alveolar NO concentration were investigated.

\section{METHODS}

\section{Subjects}

Consecutive patients attending the difficult asthma clinic at Glenfield Hospital (Leicester, UK) who fulfilled the ATS criteria for refractory asthma [1] were invited to participate. The current study also recruited volunteers with mild-to-moderate asthma and healthy volunteers who had no respiratory symptoms and normal spirometry from the present authors' research database and from local advertisement. This study was approved by the Leicestershire and Rutland ethics committee.

All subjects with asthma had symptoms consistent with asthma and at least one of the following objective measures of airway hyperresponsiveness and/or variable airflow obstruction: an increase in forced expiratory volume in one second (FEV1) of $\geqslant 15 \%$ following inhalation of $200 \mu \mathrm{g}$ of salbutamol, a peak flow amplitude as a per cent of the mean over 14 days $>20 \%$ or a methacholine provocative concentration causing a $20 \%$ fall in FEV1 (PC20) of $<8 \mathrm{mg} \cdot \mathrm{mL}^{-1}$. Refractory asthma was diagnosed according to ATS criteria, with the exception of the dose of inhaled corticosteroid, which was increased to $\geqslant 2,000 \mu \mathrm{g}$ beclomethasone equivalent $\cdot \mathrm{day}^{-1}$ to reflect the use of higher doses in UK practice; patients had at least one major criterion and two minor criteria for refractory asthma. Moderate asthma was diagnosed if no major and a maximum of one minor criterion for diagnosing refractory asthma was met and patients were on $<2,000 \mu \mathrm{g}$ of beclomethasone equivalent. Mild asthma was classified as those patients taking only a $\beta_{2}$-agonist with no minor or major criteria for refractory asthma.

Treatment concordance was assessed to be good to perfect in all patients with refractory asthma [1]. The present authors' assessment was based on measurement of serum prednisolone, cortisol and theophylline concentrations, domiciliary assessment by a consultant pharmacist and by analysis of primary care records of prescription issue and collection. Patients who were felt to be symptomatic because of uncontrolled comorbid conditions, such as rhinitis and gastro-oesophageal reflux disease, were excluded from the study.

\section{Protocol}

Exhaled NO concentration was measured at 10, 30, 50, 100 and $200 \mathrm{~mL} \cdot \mathrm{s}^{-1}$ using an online chemiluminescence analyser
(NIOX; Aerocrine, Stockholm, Sweden) prior to any other measurements. Spirometry was measured using a rolling seal spirometer (Vitalograph, Maids Moreton, UK). The methacholine PC20 was measured using the tidal breathing method, with doubling doses of inhaled methacholine inhaled though a Wright's nebuliser (Roxon, Montreal, Canada) as previously described [12]. Asthma control was assessed using the Juniper asthma control score [13]. Sputum induction was performed as previously described [14]. To assess repeatability, 25 randomly selected subjects were invited for repeated measurement of alveolar NO concentration after 2 weeks.

Patients with refractory asthma, who on clinical grounds required an increase in anti-inflammatory medication, defined as a juniper asthma control score $>1.57$ and/or a sputum eosinophil count $>3 \%$, had either oral corticosteroid treatment initiated or a doubling of their inhaled corticosteroid and were invited for repeat measurement of their alveolar NO concentration after $\geqslant 2$ weeks.

All subjects with mild asthma who were not taking inhaled corticosteroids underwent fibreoptic bronchoscopy and BAL at least 2 weeks after the initial assessment. A 20-mL aliquot of warmed sterile saline solution was instilled into the bronchus intermedius and aspirated; this was analysed as the wash sample. Three sequential samples of $60 \mathrm{~mL}$ warmed sterile saline solution were then instilled into the middle lobe bronchus and then aspirated; the pooled aspirate from these samples was analysed as the BAL fluid. The aspirates were filtered through $48 \mu \mathrm{m}$ gauze (Sefar Ltd, Bury, UK) and diluted to a cell concentration of $0.5 \times 10^{6}$ cells $\cdot \mathrm{mL}^{-1}$. Cytospins were made with $75 \mu \mathrm{L}$ of aspirate and stained with Romanowski stain prior to counting by a person blind to the subjects details. Eosinophil counts were given as a percentage of $\geqslant 400$ inflammatory cells counted.

\section{Analysis}

Alveolar NO concentration was calculated for each patient using a nonlinear model described by SILKOFF et al. [5]. Briefly, exhaled $\mathrm{NO}$ and exhalation flow were used to resolve the following nonlinear equation

$$
\mathrm{CE}=\mathrm{CW} \times(1-\exp (-\mathrm{DNO} / V))+\mathrm{CALV} \times \exp (-\mathrm{DNO} / V)
$$

In this equation, $\mathrm{CE}$ is the exhaled concentration of $\mathrm{NO}, \mathrm{CW}$ is the airway wall concentration of $\mathrm{NO}$, DNO is the diffusion constant for NO, $V$ is the exhalation flow and CALV in the alveolar NO concentration. Maximal bronchial NO output was calculated using a linear method described by TsOUKIAS and GEORGE [4], where NO output is plotted against exhalation flow and the intercept recorded as the maximal bronchial NO output. Repeatability was assessed using intraclass correlation coefficient and SD of within subject SD.

Kolmogorov-Smirnov tests of normality were performed to test the distribution of data prior to analysis. Alveolar NO concentrations were normally distributed and comparisons between groups were made using one-way ANOVA and independent sample t-tests. Alveolar NO concentration before and after oral steroid or double dose of inhaled steroids were compared with a paired t-test. Total immunoglobulin E, exhaled NO concentration, maximal bronchial NO output and sputum and BAL eosinophil counts were found to be 
Log-normally distributed and were, therefore, Log transformed prior to analysis; a value 0.1 was assigned to measurements of 0. Correlation was assessed using Pearson's product moment correlation coefficient.

\section{RESULTS}

Patient details are given in table 1 . Patients with refractory asthma had significantly lower FEV1 as a percentage of predicted (mean $62 \%$ versus $94.2 \%$, mean difference $32.2 \%$ (95\% confidence intervals (CI): 24.2-40.2); $\mathrm{p}<0.001$ ) and FEV1/ forced vital capacity ratio (mean $69 \%$ versus $78 \%$; mean difference 9.0 (95\% CI: 3.6-12.5); p<0.01) than patients with mild-to-moderate asthma. There were no significant differences between sputum eosinophil counts, single flow NO concentration, maximal bronchial NO output or other measured variables between the asthma groups (table 1).

Alveolar NO concentration measurements were possible in all patients and controls and were repeatable (intraclass correlation coefficient $=0.95$, within subject $\mathrm{SD}=0.25$ ).

There was a positive correlation between BAL eosinophil counts and alveolar NO concentration in patients with mild asthma ( $r=0.79, p=0.006$; fig. 1a) but the association between alveolar NO and bronchial wash eosinophil counts in this group was not significant $(\mathrm{r}=0.6, \mathrm{p}=0.07$; fig. $1 \mathrm{~b})$. In contrast, there was a positive association between single flow $\mathrm{NO}$ and bronchial wash eosinophil count $(r=0.69, p=0.027$; fig. $1 c)$ but no association between single flow NO and BAL eosinophil count $(r=0.25, p=0.49$; fig. $1 d)$. Similarly, bronchial NO output was positively correlated with bronchial wash eosinophil count $(\mathrm{r}=0.71, \mathrm{p}=0.022)$ but not with BAL eosinophil count $(\mathrm{r}=0.1, \mathrm{p}=0.76)$. Sputum eosinophil count was positively correlated with single flow NO $(r=0.7, p=0.02)$ and bronchial NO $(\mathrm{r}=0.82, \mathrm{p}=0.004)$ but not alveolar $\mathrm{NO}(\mathrm{r}=0.2$, $\mathrm{p}=0.6)$.
The mean \pm SEM alveolar NO concentration was $7.1 \pm 0.70 \mathrm{ppb}$ in the refractory asthma group, $3.4 \pm 0.46 \mathrm{ppb}$ in the mild-tomoderate asthma group and $3.4 \pm 0.38 \mathrm{ppb}$ in the normal controls $(p<0.001)$. There were significant differences in alveolar NO concentration between refractory asthma and normal controls (difference $3.7 \mathrm{ppb}$ (95\% CI: 1.59-5.83); $\mathrm{p}=0.001$ ) and mild-to-moderate asthma (difference $3.7 \mathrm{ppb}$ (95\% CI: 2.0-5.4); $\mathrm{p}<0.001)$ but not between normal controls and mild-to-moderate asthma (fig. 2).

Within the mild-to-moderate asthma group there was no difference in alveolar NO concentration between the steroid naive and inhaled steroid treated group (3.6 $\mathrm{ppb}$ versus $3.4 \mathrm{ppb} ; \mathrm{p}=0.82)$. Within the refractory asthma group there was no significant difference in the concentration of alveolar NO in subjects receiving oral corticosteroids and those who were not. There was no relationship between alveolar NO and the dose or type of inhaled corticosteroid used, no significant difference in alveolar NO concentration between eosinophilic and noneosinophilic asthma and no relationship with Juniper asthma control score. There was no difference in alveolar NO concentration between groups within the refractory asthma group, defined according to the most prominent secondary feature of refractory asthma. There was a weak negative correlation between alveolar NO concentration and postbronchodilator FEV1 \% predicted $(\mathrm{r}=-0.28 ; \mathrm{p}=0.017)$.

The baseline concentration of alveolar NO was not significantly different in the patients who were started on oral corticosteroids (mean $7.9 \mathrm{ppb}$ ) than those who received a doubling dose of their inhaled corticosteroid (mean $7.5 \mathrm{ppb}$, mean difference 0.4 (95\% CI: $-3.9-2.5) ; p=0.65)$. Other baseline characteristics were similar (table 2). Treatment with oral corticosteroids led to a significant reduction in alveolar NO concentration (7.9-3.6 ppb, mean difference 4.3 (95\% CI: $1.3-$ $7.4) ; \mathrm{p}=0.002)$, whereas a doubling of the dose of inhaled

\section{TABLE 1 Patient demographics}

\begin{tabular}{|c|c|c|c|}
\hline & Normal & Mild-to-moderate asthma & Refractory asthma \\
\hline Subjects $n$ & 13 & 25 & 27 \\
\hline Atopic \% & $3 \pm 23$ & $11 \pm 44$ & $14 \pm 52$ \\
\hline Total $\lg \mathrm{E}^{\#}$ & $16.2(0.6)$ & $172(1.1)$ & $354(0.6)$ \\
\hline Age yrs & $38(23-49)$ & $42(18-72)$ & $44(21-69)$ \\
\hline Nocturnal symptoms $n$ & 0 & 6 & 10 \\
\hline FEV $1 \%$ pred & $96.6 \pm 5.9$ & $94.2 \pm 7.4$ & $62 \pm 21.1$ \\
\hline FEV $1 / F V C \%$ & $79.6 \pm 7.8$ & $78.0 \pm 6.7$ & $69.0 \pm 13$ \\
\hline Single flow NO concentration $\mathrm{ppb}, 50 \mathrm{~mL} \cdot \mathrm{s}^{-1 \#}$ & $16.5(0.18)$ & $24.6(0.31)$ & $31.7(0.28)$ \\
\hline Maximal bronchial $\mathrm{NO}$ output $\mathrm{nL} \cdot \mathrm{min}^{-1 \#}$ & $39.3(0.2)$ & $54.3(0.4)$ & $64.3(0.4)$ \\
\hline Sputum eosinophil count $\%^{+}$ & $0(0-1.3)$ & $6.3(0-65.8)$ & $7.8(0-53.3)$ \\
\hline
\end{tabular}

Data are presented as mean \pm SD, unless otherwise indicated. Ig: immunoglobulin; FEV1: forced expiratory volume in one second; \% pred: per cent predicted; FVC: forced vital capacity; NO: nitric oxide; PC20: provocative concentration causing a $20 \%$ fall in FEV1; NA: not available. \#: geometric mean (log sD); ${ }^{\uparrow}$ : mean (range); ${ }^{+}$: median (range). 

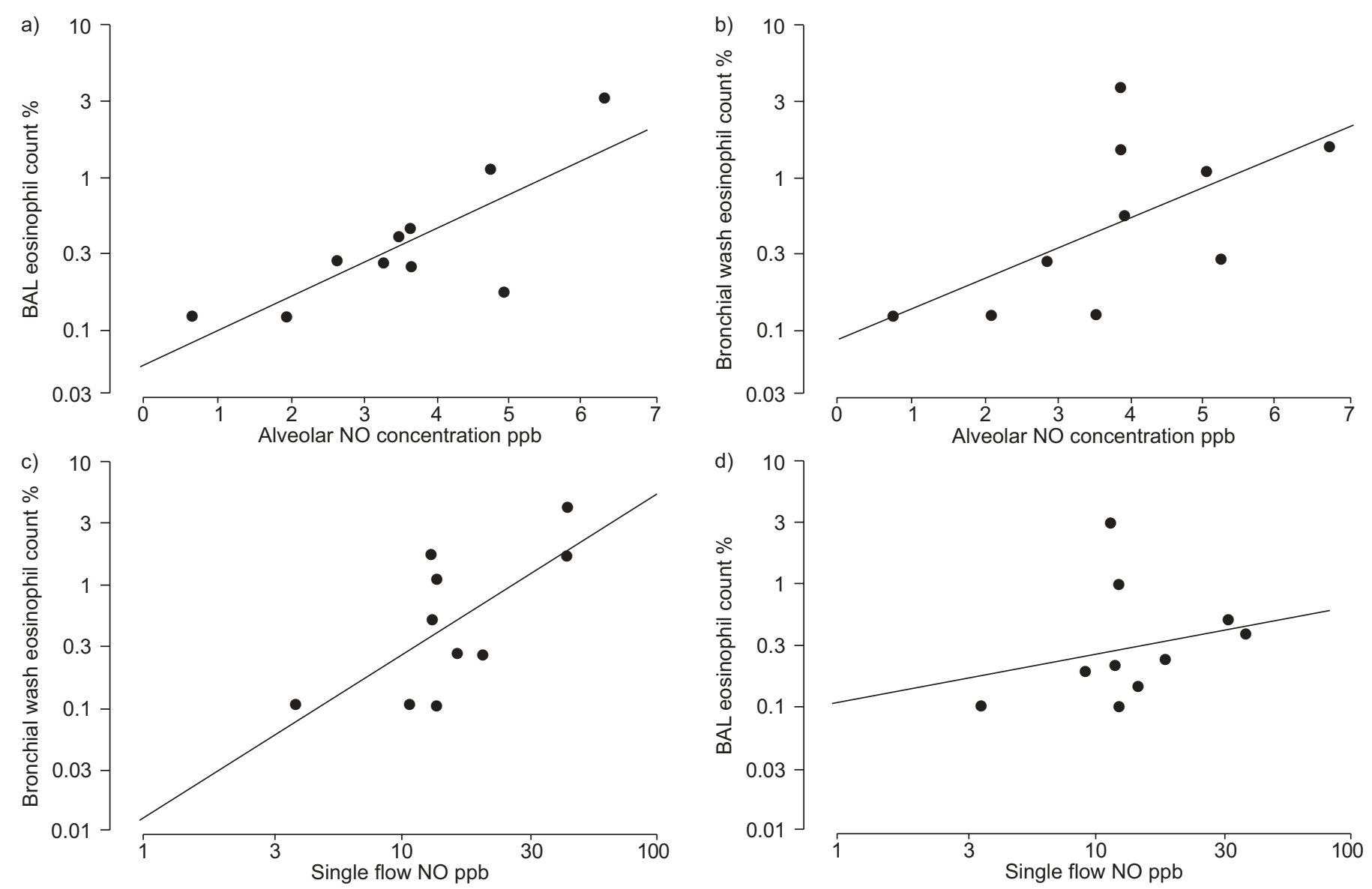

FIGURE 1. Association between a) bronchoalveolar lavage (BAL) eosinophils and alveolar nitric oxide (NO) concentration ( $r=0.79, p=0.006$ ), $b$ ) bronchial wash eosinophils and alveolar NO concentration ( $r=0.6, p=0.07)$, c) bronchial wash eosinophil count and single flow NO in patients with mild steroid-naive asthma ( $r=0.69$, $p=0.027)$ and $d)$ single flow $N O$ and BAL eosinophil count $(r=0.25, p=0.49)$.

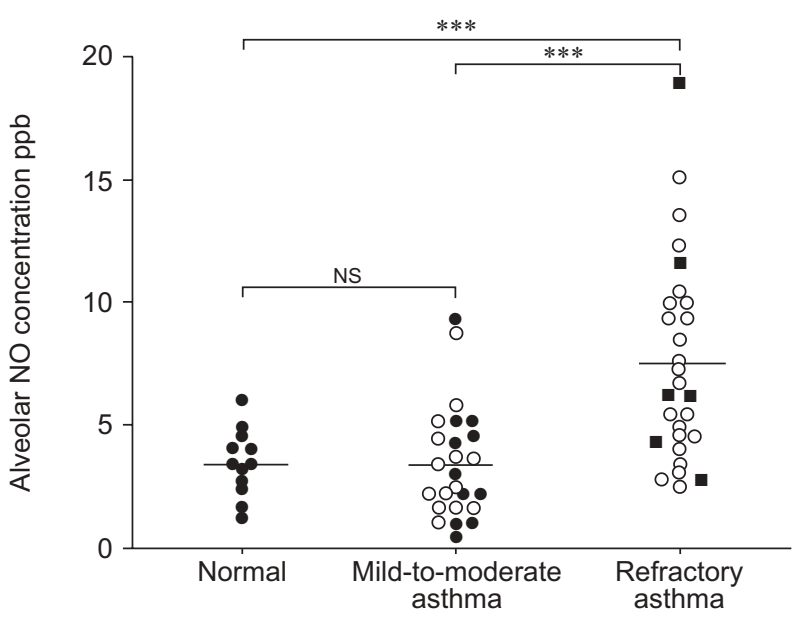

FIGURE 2. Alveolar nitric oxide (NO) concentration in normal controls compared to mild-to-moderate asthma and refractory asthma. $\bullet$ : steroid naive; $\mathrm{O}$ : inhaled steroid treated only; $\mathbf{\square}$ : oral and inhaled steroid treated. ${ }^{* *}$ : $p<0.001$ NS: nonsignificant.

corticosteroids did not lead to a reduction $(7.5-8.0 \mathrm{ppb}$, mean difference -0.6 (95\% CI: $-3.2-2) ; \mathrm{p}=0.62 ;$ fig. 3). The difference in the change in alveolar NO concentration between the two treatment groups was significant (mean difference $5.0(95 \% \mathrm{CI}$ : $1.0-5.0) ; \mathrm{p}=0.034)$.

\section{DISCUSSION}

The present study found that measurement of alveolar NO concentration was feasible in patients with refractory asthma and that results are repeatable over 2 weeks. Alveolar NO concentration correlated more closely with BAL than bronchial wash eosinophil cell counts, whereas the reverse was true with single flow NO concentration. Bronchial NO output, a measure of NO flux, was also related to sputum and bronchial wash eosinophil counts but not BAL cells. Increased alveolar NO was seen in patients with refractory asthma who were already receiving highdose inhaled steroids. Finally, a 2-week course of oral prednisolone, but not a 1-month course of double the dose of inhaled corticosteroids, reduced alveolar NO concentration in patients who required a step-up in their treatment. These findings support the current authors' hypothesis that alveolar NO is a measure of inflammation in the distal lung and are consistent with the view that refractory asthma is associated with distal lung inflammation. The present study suggests that inflammation in this site responds to oral but not inhaled corticosteroids. 


\begin{tabular}{|c|c|c|c|c|c|c|c|c|}
\hline & \multicolumn{2}{|c|}{ Oral corticosteroids } & Difference & p-value & \multicolumn{2}{|c|}{ Inhaled corticosteroids } & Difference & p-value \\
\hline Alveolar NO ppb & $7.9 \pm 1.4$ & $3.58 \pm 0.5$ & $4.3(1.3-7.4)$ & 0.008 & $7.48 \pm 1.6$ & $8.0 \pm 1.9$ & $0.6(-2-3.2)$ & \\
\hline Single flow NO ppb & $32.4 \pm 0.1^{\#}$ & $17.8 \pm 0.1^{\#}$ & $1.8(0.8-4.4)^{\circ}$ & 0.16 & $41.6 \pm 0.1^{\#}$ & $41.3 \pm 0.1^{\#}$ & $1.0(0.8-1.4)^{\bullet}$ & 0.897 \\
\hline Juniper asthma control score & $2.8 \pm 0.6$ & $1.6 \pm 0.2$ & $1.2(0.01-2.3)$ & 0.036 & $2.6 \pm 0.3$ & $1.8 \pm 0.5$ & $0.8(-0.04-1.7)$ & 0.057 \\
\hline Post-bronchodilator FEV $1 \%$ pred & $64 \pm 8.6$ & $78 \pm 5.4$ & $14(2-26)$ & 0.027 & $73 \pm 5.2$ & $77 \pm 4.8$ & $4.2(-3.9-12.4)$ & 0.259 \\
\hline $\mathrm{FEV}_{1 / \mathrm{FVC}}$ ratio $\%$ & $74 \pm 2.8$ & $76 \pm 3.0$ & $2.2(0.4-4.8)$ & 0.086 & $71 \pm 4$ & $72 \pm 2.4$ & $0.7(-7-8)$ & 0.844 \\
\hline
\end{tabular}

Data are presented as mean \pm SE and differences are presented as difference ( $95 \%$ confidence intervals), unless otherwise indicated. NO: nitric oxide; FEV 1 : forced expiratory volume in one second; \% pred: per cent predicted; FVC: forced vital capacity. \#: geometric mean \pm log SE; " : fold difference (95\% confidence intervals).

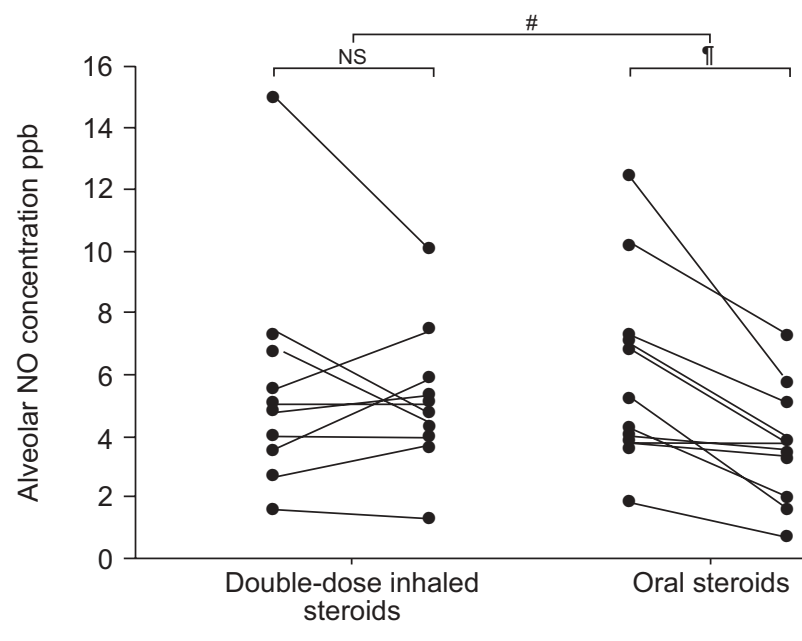

FIGURE 3. Changes in alveolar nitric oxide (NO) concentration in patients who received a doubling of their inhaled steroid dose $(n=10)$ and those who received oral corticosteroids $(n=11){ }^{\#}: p=0.034 ;{ }^{~}$ : $p=0.002$; NS: nonsignificant.

Bronchoscopy and BAL is a relatively invasive procedure, so this study was limited to studying the relationship between alveolar NO and BAL eosinophils in subjects with mild asthma. Alveolar NO concentrations were normal in this population and there was limited inter-individual variability in alveolar NO concentration. Despite these limitations, a strong positive correlation between alveolar NO and BAL eosinophil count was found but not with bronchial wash eosinophils. The opposite was true for single flow $\mathrm{NO}$ and bronchial $\mathrm{NO}$ output. A previous study highlighted the weak relationship between BAL and sputum cell counts and has suggested that this is due to sampling of different compartments, with BAL accessing the distal lung and sputum the proximal airway [9]. The current authors' finding of a strong positive correlation between BAL eosinophils and alveolar $\mathrm{NO}$ and the previous observation in children with asthma that alveolar NO and BAL ECP are correlated may therefore be consistent with the view that elevated alveolar NO reflects eosinophilic inflammation in the distal lung.

The current authors have shown that alveolar NO concentration is higher in patients with refractory asthma than in both normal controls and patients with mild-to-moderate asthma. There was no difference in proximal airway inflammation assessed by sputum eosinophil count, single flow NO concentration or bronchial $\mathrm{NO}$ output, implying that the difference in alveolar NO concentration is due to the difference in the site rather than the intensity of the lower airway response. Nocturnal wheeze was reported by a similar proportion of patients in all asthma groups so the current authors' findings are unlikely to be due to differences in the prevalence of nocturnal asthma, a condition that has been associated with high alveolar NO concentrations [15]. Similarly, there was no relationship between asthma control, assessed using the Juniper asthma control score, at the time of sampling and alveolar NO concentration, suggesting that distal airway inflammation occurs in patients who require high doses of inhaled steroids or oral steroids to achieve control of their symptoms, as well as patients with uncontrolled asthma.

The presence of inflammation in the distal lung suggests a possible mechanism for inhaled corticosteroid resistance, the characteristic feature of refractory asthma, since the distal lung might not be accessed by inhaled corticosteroids that preferentially deposit in the larger airways [16]. The concept that distal lung inflammation is not modified by inhaled corticosteroids is supported by the presence of high alveolar NO in patients who were taking high dose inhaled corticosteroids and by an earlier study, in mild-to-moderate asthma, showing that inhaled fluticasone does not reduce alveolar NO [8]. Further support for this view and evidence that even elevated alveolar NO concentrations cannot be modified by inhaled corticosteroids comes from the current authors observation that a double dose of inhaled corticosteroid did not reduce alveolar NO concentration. By contrast, oral prednisolone significantly reduced alveolar NO. The clear implication of this finding is that alveolar $\mathrm{NO}$ concentration reflects inflammation in a site that can be accessed by systemic but not inhaled corticosteroids.

An important limitation of the current investigation of the effect of oral and inhaled corticosteroids on alveolar NO concentration is that patients were not randomised to the different treatments and the interventions were not placebo controlled. However, patients who received prednisolone or higher dose inhaled corticosteroids had similar pre-treatment 
characteristics so the current findings are unlikely to be biased by differences in severity of disease. The present authors also doubt that the reduction in alveolar NO concentration seen with prednisolone was due to regression to the mean, since a similar reduction was not seen with higher dose inhaled steroids. Nevertheless, the present authors recognise that further randomised placebo-controlled studies are required to investigate the effects of oral and inhaled corticosteroids on alveolar NO concentration before the findings from the current study can be regarded as definitive.

There is increasing recognition that refractory asthma is a heterogeneous disease [1, 2]. Studies have highlighted the presence of noneosinophilic pathology in some patients [17]. Others have identified fixed airflow obstruction [18] or recurrent exacerbation [14] as important phenotypes. Implicit in the definition is the concept that asthma control cannot be achieved with inhaled corticosteroids or that high dose treatment is required [1, 2]. In the current population increased alveolar NO concentration appeared to be a feature of patients with different criteria for a diagnosis of refractory asthma, perhaps consistent with the view that distal lung inflammation is a common feature in different types of refractory asthma. However, the current authors recognise that the power of the current study to identify a difference is limited and further work is required to investigate this important question. One intriguing observation was the significant correlation between post-bronchodilator FEV1 and alveolar NO concentration, suggesting that distal lung inflammation might be involved in the development of fixed airflow obstruction in asthma.

In conclusion, this preliminary study suggests that alveolar nitric oxide is a potentially useful technique for investigating the role of distal lung inflammation in asthma and other airway diseases. The findings are consistent with the presence of distal lung inflammation in refractory asthma and suggest that eosinophilic inflammation in this site might be associated with inhaled but not systemic corticosteroid resistance.

\section{ACKNOWLEDGEMENTS}

The authors would like to thank W. Monteiro for staining and counting the cytospins of BAL and bronchial wash.

\section{REFERENCES}

1 Proceedings of the ATS workshop on refractory asthma: current understanding, recommendations, and unanswered questions. American Thoracic Society. Am J Respir Crit Care Med 2000; 162: 2341-2351.

2 Chung KF, Godard P, Adelroth E, et al. Difficult/therapyresistant asthma: the need for an integrated approach to define clinical phenotypes, evaluate risk factors, understand pathophysiology and find novel therapies. Eur Respir J 1999; 13: 1198-1208.

3 Kraft M, Djukanovic R, Wilson S, Holgate ST, Martin RJ. Alveolar tissue inflammation in asthma. Am J Respir Crit Care Med 1996; 154: 1505-1510.
4 Tsoukias NM, George SC. A two-compartment model of pulmonary nitric oxide exchange dynamics. J Appl Physiol 1998; 85: 653-666.

5 Silkoff PE, Sylvester JT, Zamel N, Permutt S. Airway nitric oxide diffusion in asthma: role in pulmonary function and bronchial responsiveness. Am J Respir Crit Care Med 2000; 161: 1218-1228.

6 Lehtimaki L, Kankaanranta H, Saarelainen S, et al. Extended exhaled NO measurement differentiates between alveolar and bronchial inflammation. Am J Respir Crit Care Med 2001; 163: 1557-1561.

7 Hogman M, Holmkvist T, Wegener T, et al. Extended NO analysis applied to patients with COPD, allergic asthma and allergic rhinitis. Respir Med 2002; 96: 24-30.

8 Lehtimaki L, Kankaanranta H, Saarelainen S, Turjanmaa V, Moilanen E. Inhaled fluticasone decreases bronchial but not alveolar nitric oxide output in asthma. Eur Respir J 2001; 18: 635-639.

9 Pizzichini E, Pizzichini MM, Kidney JC, et al. Induced sputum, bronchoalveolar lavage and blood from mild asthmatics: inflammatory cells, lymphocyte subsets and soluble markers compared. Eur Respir J 2004; 11: 828-834.

10 Sutherland ER, Martin RJ, Bowler RP, et al. Physiologic correlates of distal lung inflammation in asthma. J Allergy Clin Immunol 2004; 113: 1046-1050.

11 Mahut B, Delclaux C, Tillie-Leblond I, et al. Both inflammation and remodeling influence nitric oxide output in children with refractory asthma. J Allergy Clin Immunol 2004; 113: 252-256.

12 Juniper EF, Cockcroft DW, Hargreave FE. Histamine and methacholine inhalation tests: a laboratory tidal breathing protocol. Lund, Sweden, Astra Draco Ltd, 1994.

13 Juniper EF, O'Byrne PM, Ferrie PJ, King DR, Roberts JN. Measuring asthma control. Clinic questionnaire or daily diary? Am J Respir Crit Care Med 2000; 162: 1330-1334.

14 Green RH, Brightling CE, McKenna S, et al. Asthma exacerbations and sputum eosinophil counts: a randomised controlled trial. Lancet 2002; 360: 1715-1721.

15 Lehtimaki L, Kankaanranta H, Saarelainen S, Turjanmaa V, Moilanen E. Increased alveolar nitric oxide concentration in asthmatic patients with nocturnal symptoms. Eur Respir J 2002; 20: 841-845.

16 Martin RJ. Therapeutic significance of distal airway inflammation in asthma. J Allergy Clin Immunol 2002; 109: Suppl. 2, S447-S460.

17 Wenzel SE, Schwartz LB, Langmack EL, et al. Evidence that severe asthma can be divided pathologically into two inflammatory subtypes with distinct physiologic and clinical characteristics. Am J Respir Crit Care Med 1999; 160: 1001-1008.

18 ten Brinke A, Zwinderman AH, Sterk PJ, Rabe KF, Bel EH. Factors associated with persistent airflow limitation in severe asthma. Am J Respir Crit Care Med 2001; 164: 744-748. 